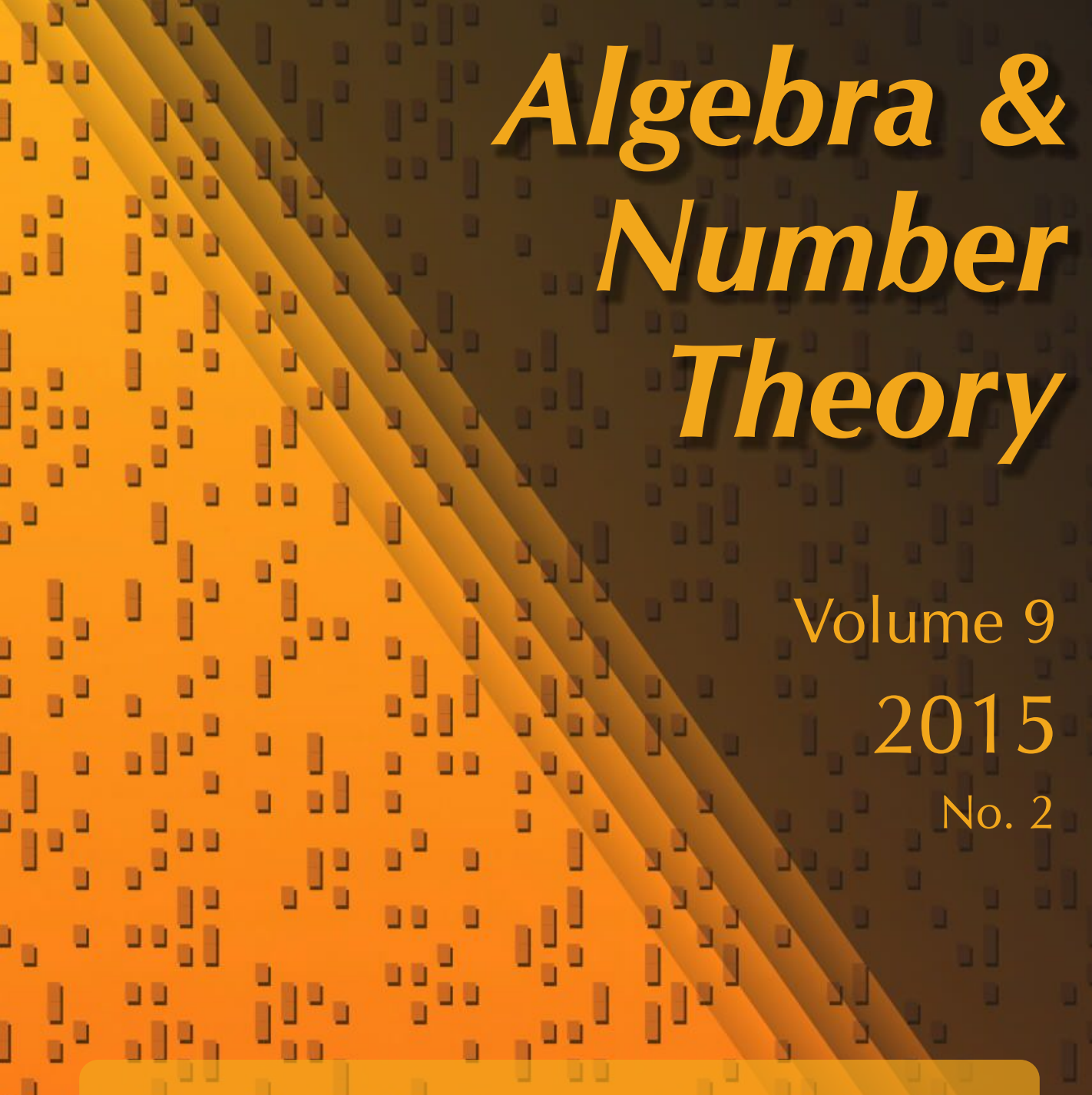

Semiample invertible sheaves with semipositive continuous hermitian metrics

Atsushi Moriwaki

\lrcorner

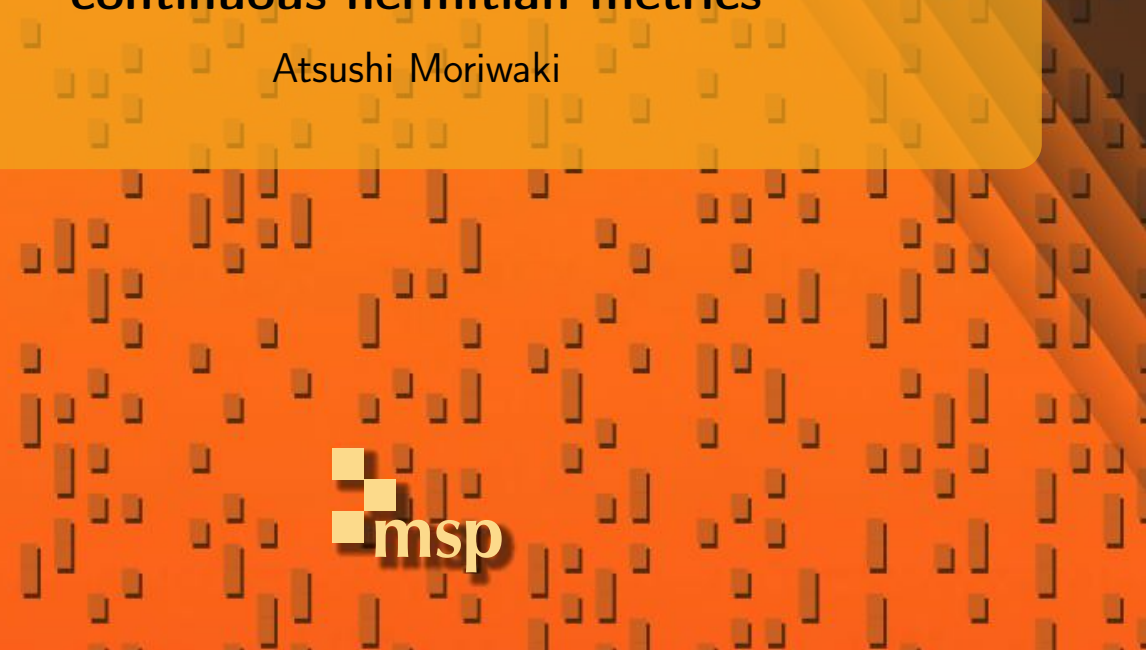




\title{
Semiample invertible sheaves with semipositive continuous hermitian metrics
}

\author{
Atsushi Moriwaki
}

\begin{abstract}
Let $(L, h)$ be a pair of a semiample invertible sheaf and a semipositive continuous hermitian metric on a proper algebraic variety over $\mathbb{C}$. In this paper, we prove that $(L, h)$ is semiample metrized, answering a generalization of a question of S. Zhang.
\end{abstract}

\section{Introduction}

Let $X$ be a proper algebraic variety over $\mathbb{C}$. Let $L$ be an invertible sheaf on $X$, and let $h$ be a continuous hermitian metric of $L$. We say that $(L, h)$ is semiample metrized if, for any $\epsilon>0$, there is $n>0$ such that, for any $x \in X(\mathbb{C})$, we can find $l \in H^{0}\left(X, L^{\otimes n}\right) \backslash\{0\}$ with

$$
\sup \left\{h^{\otimes n}(l, l)(w) \mid w \in X(\mathbb{C})\right\} \leq e^{\epsilon n} h^{\otimes n}(l, l)(x) .
$$

Shouwu Zhang proposed the following question:

Question 0.1 [Zhang 1995, Question 3.6]. If $L$ is ample and $h$ is smooth and semipositive, does it follow that $(L, h)$ is semiample metrized?

Theorem 3.5 of the same reference gives an affirmative answer in the case where $X$ is smooth over $\mathbb{C}$. The purpose of this paper is to give an answer for a generalization of the above question. First of all, we fix some notation: We say that $L$ is semiample if there is a positive integer $n_{0}$ such that $L^{\otimes n_{0}}$ is generated by global sections. Moreover, $h$ is said to be semipositive (or we say that $(L, h)$ is semipositive) if, for any point $x \in X(\mathbb{C})$ and a local basis $s$ of $L$ on a neighborhood of $x$, $-\log h(s, s)$ is plurisubharmonic around $x$ (for the definition of plurisubharmonicity on a singular variety, see Section 1). Note that $h$ is not necessarily smooth. By using the recent work of Coman, Guedj and Zeriahi [Coman et al. 2013], we have the following answer:

Theorem 0.2. If $L$ is semiample and $h$ is continuous and semipositive, then $(L, h)$ is semiample metrized.

MSC2010: primary 14C20; secondary 32U05, 14G40.

Keywords: semiample metrized, semipositive. 


\section{Plurisubharmonic functions on singular complex analytic spaces}

Let $T$ be a reduced complex analytic space. An upper-semicontinuous function

$$
\varphi: T \rightarrow \mathbb{R} \cup\{-\infty\}
$$

is said to be plurisubharmonic if $\varphi \not \equiv-\infty$ and, for each $x \in T$, there is an analytic closed embedding $\iota_{x}: U_{x} \hookrightarrow W_{x}$ of an open neighborhood $U_{x}$ of $x$ into an open set $W_{x}$ of $\mathbb{C}^{n_{x}}$ together with a plurisubharmonic function $\Phi_{x}$ on $W_{x}$ such that $\left.\varphi\right|_{U_{x}}=\iota_{x}^{*}\left(\Phi_{x}\right)$. For an analytic map $f: T^{\prime} \rightarrow T$ of reduced complex analytic spaces and a plurisubharmonic function $\varphi$ on $T$, it is easy to see that $\varphi \circ f$ is either identically $-\infty$ or plurisubharmonic on $T^{\prime}$. By [Fornæss and Narasimhan 1980, Theorem 5.3.1], an upper-semicontinuous function $\varphi: T \rightarrow \mathbb{R} \cup\{-\infty\}$ is plurisubharmonic if and only if, for any analytic map $\varrho: \mathbb{D} \rightarrow T, \varphi \circ \varrho$ is either identically $-\infty$ or subharmonic on $\mathbb{D}$, where $\mathbb{D}:=\{z \in \mathbb{C}|| z \mid<1\}$. Moreover, if $T$ is compact and $\varphi$ is plurisubharmonic on $T$, then $\varphi$ is locally constant.

Let $\omega$ be a smooth $(1,1)$-form on $T$, that is, in the same way as in the definition of plurisubharmonic functions, $\omega$ is a smooth $(1,1)$-form on the regular part of $T$ and, for each $x \in T$, there is an analytic closed embedding $\iota_{x}: U_{x} \hookrightarrow W_{x}$ of an open neighborhood $U_{x}$ of $x$ into an open set $W_{x}$ of $\mathbb{C}^{n_{x}}$ together with a smooth $(1,1)$-form $\Omega_{x}$ on $W_{x}$ such that $\left.\omega\right|_{U_{x}}=\iota_{x}^{*}\left(\Omega_{x}\right)$. We assume that $\omega$ is locally given by $d d^{c}(u)$ for some smooth function $u$ on a neighborhood of $x$. Let $\phi$ be a quasiplurisubharmonic function on $T$; that is, for each $x \in T, \phi$ can be locally written as the sum of a smooth function and a plurisubharmonic function around $x$. We say that $\phi$ is $\omega$-plurisubharmonic if there is an open covering $T=\bigcup_{\lambda} U_{\lambda}$, together with a smooth function $u_{\lambda}$ on $U_{\lambda}$ for each $\lambda$, such that $\left.\omega\right|_{U_{\lambda}}=d d^{c}\left(u_{\lambda}\right)$ and $\left.\phi\right|_{U_{\lambda}}+u_{\lambda}$ is plurisubharmonic on $U_{\lambda}$. The condition for $\omega$-plurisubharmonicity is often denoted by $d d^{c}([\phi])+\omega \geq 0$.

Here we consider the following lemma:

Lemma 1.1. Let $f: X \rightarrow Y$ be a surjective and proper morphism of algebraic varieties over $\mathbb{C}$. Let $\varphi$ be a real-valued function on $Y(\mathbb{C})$.

(1) $\varphi$ is continuous if and only if $\varphi \circ f$ is continuous.

(2) Assume that $\varphi$ is continuous. Then $\varphi$ is plurisubharmonic if and only if $\varphi \circ f$ is plurisubharmonic.

Proof. (1) It is sufficient to see that if $\varphi \circ f$ is continuous, then $\varphi$ is continuous. Otherwise, there are $y \in Y(\mathbb{C}), \epsilon_{0}>0$ and a sequence $\left\{y_{n}\right\}$ on $Y(\mathbb{C})$ such that $\lim _{n \rightarrow \infty} y_{n}=y$ and $\left|\varphi\left(y_{n}\right)-\varphi(y)\right| \geq \epsilon_{0}$ for all $n$. We choose $x_{n} \in X(\mathbb{C})$ such that $f\left(x_{n}\right)=y_{n}$. As $f: X \rightarrow Y$ is proper, we can find a subsequence $\left\{x_{n_{i}}\right\}$ of $\left\{x_{n}\right\}$ such that $x:=\lim _{i \rightarrow \infty} x_{n_{i}}$ exists in $X(\mathbb{C})$. Note that

$$
f(x)=\lim _{i \rightarrow \infty} f\left(x_{n_{i}}\right)=\lim _{i \rightarrow \infty} y_{n_{i}}=y,
$$


so that, as $\varphi \circ f$ is continuous,

$$
\varphi(y)=(\varphi \circ f)(x)=\lim _{i \rightarrow \infty}(\varphi \circ f)\left(x_{n_{i}}\right)=\lim _{i \rightarrow \infty} \varphi\left(f\left(x_{n_{i}}\right)\right)=\lim _{i \rightarrow \infty} \varphi\left(y_{n_{i}}\right),
$$

which is a contradiction, so that $\varphi$ is continuous.

(2) We need to check that if $\varphi \circ f$ is plurisubharmonic, then $\varphi$ is plurisubharmonic. By using Chow's lemma, we may assume that $f: X \rightarrow Y$ is projective. Moreover, since the assertion is local with respect to $Y$, we may further assume that there is a closed embedding $\iota: X \hookrightarrow Y \times \mathbb{P}^{N}$ such that $p \circ \iota=f$, where $p: Y \times \mathbb{P}^{n} \rightarrow Y$ is the projection to the first factor. The remaining proof is same as the last part of the proof of [Demailly 1985, Theorem 1.7]. Let $g:(\mathbb{D}, 0) \rightarrow(Y, y)$ be a germ of an analytic map. By the theorem of Fornæss and Narasimhan, it is sufficient to show that $\varphi \circ g$ is subharmonic. Clearly we may assume that $g$ is given by the normalization of a 1-dimensional irreducible germ $(C, y)$ in $(Y, y)$. Using hyperplanes in $\mathbb{P}^{N}$, we can find $x \in X$ and a 1-dimensional irreducible germ $\left(C^{\prime}, x\right)$ in $(X, x)$ such that $\left(C^{\prime}, x\right)$ lies over $(C, y)$. Let $g^{\prime}:(\mathbb{D}, 0) \rightarrow(X, x)$ be the germ of an analytic map given by the normalization of $\left(C^{\prime}, x\right)$. Then we have an analytic map $\sigma:(\mathbb{D}, 0) \rightarrow(\mathbb{D}, 0)$ with $g \circ \sigma=f \circ g^{\prime}$ :

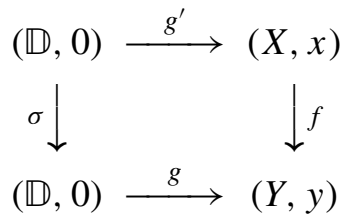

Changing a variable of $(\mathbb{D}, 0)$, we may assume that $\sigma$ is given by $\sigma(z)=z^{m}$ for some positive integer $m$. Then $\varphi \circ g \circ \sigma$ is subharmonic because $\varphi \circ f$ is plurisubharmonic. Therefore, as $\sigma$ is étale over the outside of $0, \varphi \circ g$ is subharmonic on the outside of 0 , and hence $\varphi \circ g$ is subharmonic on $(\mathbb{D}, 0)$ by the removability of singularities of subharmonic functions.

\section{Descent of a semipositive continuous hermitian metric}

Here, we consider a descent problem of a semipositive continuous hermitian metric.

Theorem 2.1. Let $f: X \rightarrow Y$ be a surjective and proper morphism of algebraic varieties over $\mathbb{C}$ with $f_{*} O_{X}=O_{Y}$. Let $L$ be an invertible sheaf on $Y$. If $h^{\prime}$ is a semipositive continuous hermitian metric of $f^{*}(L)$, then there is a semipositive continuous hermitian metric $h$ of $L$ such that $h^{\prime}=f^{*}(h)$.

Proof. Let $h_{0}$ be a continuous hermitian metric of $L$ on $Y$. There is a continuous function $\phi$ on $X(\mathbb{C})$ such that $h^{\prime}=\exp (\phi) f^{*}\left(h_{0}\right)$. Let $F$ be a subvariety of $X$ such that $F$ is an irreducible component of a fiber of $f: X \rightarrow Y$. Then, as

$$
\left.\left(f^{*}(L), h^{\prime}\right)\right|_{F} \simeq\left(\bigcirc_{F}, \exp \left(\left.\phi\right|_{F}\right)\right),
$$


we can see that $-\left.\phi\right|_{F}$ is plurisubharmonic, so that $\left.\phi\right|_{F}$ is constant. Therefore, for any point $y \in Y(\mathbb{C}),\left.\phi\right|_{\mu^{-1}(y)}$ is constant because $\mu^{-1}(y)$ is connected, and hence there is a function $\psi$ on $Y(\mathbb{C})$ such that $\psi \circ f=\phi$. By Lemma 1.1(1), $\psi$ is continuous, so that, if we set $h:=\exp (\psi) h_{0}$, then $h$ is continuous on $Y(\mathbb{C})$ and $h^{\prime}=f^{*}(h)$.

Finally, let us see that $h$ is semipositive. As this is a local question on $Y$, we may assume that there is a local basis $s$ of $L$ over $Y$. If we set $\varphi=-\log h(s, s)$, then $\varphi \circ f$ is plurisubharmonic because $h^{\prime}$ is semipositive. Therefore, by Lemma 1.1(2), $\varphi$ is plurisubharmonic, as required

\section{The proof of Theorem 0.2}

In the case where $X$ is smooth over $\mathbb{C}, L$ is ample and $h$ is smooth, this theorem was proved by Zhang [1995, Theorem 3.5]. First we assume that $L$ is ample. Then there are a positive integer $n_{0}$ and a closed embedding $X \hookrightarrow \mathbb{P}^{N}$ such that $\left.\mathbb{O}_{\mathbb{P}^{N}}(1)\right|_{X} \simeq L^{\otimes n_{0}}$. Let $h_{\mathrm{FS}}$ be the Fubini-Study metric of $\mathcal{O}_{\mathbb{P}^{n}}(1)$. Let $\phi$ be the continuous function on $X(\mathbb{C})$ given by $h^{\otimes n_{0}}=\left.\exp (-\phi) h_{\mathrm{FS}}\right|_{X}$. We set $\omega=c_{1}\left(\mathcal{O}_{\mathbb{P}^{N}}(1), h_{\mathrm{FS}}\right)$. Then $\phi$ is $\left(\left.\omega\right|_{X}\right)$-plurisubharmonic. Therefore, by [Coman et al. 2013, Corollary $\mathbb{C}$ ], there is a sequence $\left\{\varphi_{i}\right\}$ of smooth functions on $\mathbb{P}^{N}(\mathbb{C})$ with the following properties:

(1) $\varphi_{i}$ is $\omega$-plurisubharmonic for all $i$.

(2) $\varphi_{i} \geq \varphi_{i+1}$ for all $i$.

(3) For $x \in X(\mathbb{C}), \lim _{i \rightarrow \infty} \varphi_{i}(x)=\phi(x)$.

Since $X$ is compact and $\phi$ is continuous, (3) implies that the sequence $\left\{\varphi_{i}\right\}$ converges to $\phi$ uniformly on $X(\mathbb{C})$. We choose $i$ such that $\left|\phi(x)-\varphi_{i}(x)\right| \leq \epsilon n_{0} / 2$ for all $x \in X$. We set $h_{i}=\exp \left(-\varphi_{i}\right) h_{\mathrm{FS}}$. Then $h_{i}$ is a semipositive smooth hermitian metric of $O_{\mathbb{P}^{N}}(1)$. Therefore, there is a positive integer $n_{1}$ such that, for $x \in \mathbb{P}^{N}(\mathbb{C})$, we can find $l \in H^{0}\left(\mathbb{P}^{N}, \mathscr{O}_{\mathbb{P}^{N}}\left(n_{1}\right)\right) \backslash\{0\}$ with

$$
\sup \left\{h_{i}^{\otimes n_{1}}(l, l)(w) \mid w \in \mathbb{P}^{N}(\mathbb{C})\right\} \leq e^{n_{1}\left(\epsilon n_{0} / 2\right)} h_{i}^{\otimes n_{1}}(l, l)(x) .
$$

In particular, if $x \in X(\mathbb{C})$, then $l(x) \neq 0$ (so that $\left.l\right|_{X} \neq 0$ ) and

$$
\sup \left\{h_{i}^{\otimes n_{1}}(l, l)(w) \mid w \in X(\mathbb{C})\right\} \leq e^{\epsilon n_{0} n_{1} / 2} h_{i}^{\otimes n_{1}}(l, l)(x) .
$$

Note that

$$
h^{\otimes n_{0}} e^{-\epsilon n_{0} / 2} \leq h_{i} \leq h^{\otimes n_{0}}
$$

on $X(\mathbb{C})$, because $h_{i}=h^{\otimes n_{0}} \exp \left(\phi-\varphi_{i}\right)$ and $-\epsilon n_{0} / 2 \leq \phi-\varphi_{i} \leq 0$ on $X(\mathbb{C})$. Therefore,

$$
\sup \left\{h^{\otimes n_{0} n_{1}}(l, l)(w) \mid w \in X(\mathbb{C})\right\} e^{-n_{0} n_{1} \epsilon / 2} \leq \sup \left\{h_{i}^{\otimes n_{1}}(l, l)(w) \mid w \in X(\mathbb{C})\right\}
$$


and

$$
h_{i}^{\otimes n_{1}}(l, l)(x) \leq h^{\otimes n_{0} n_{1}}(l, l)(x),
$$

and hence

$$
\sup \left\{h^{\otimes n_{0} n_{1}}(l, l)(w) \mid w \in X(\mathbb{C})\right\} \leq e^{n_{1} n_{0} \epsilon} h^{\otimes n_{0} n_{1}}(l, l)(x),
$$

as required.

In general, as $L$ is semiample, there are a positive integer $n_{2}$, a projective algebraic variety $Y$ over $\mathbb{C}$, a morphism $f: X \rightarrow Y$ and an ample invertible sheaf $A$ on $Y$ such that $f_{*} \mathcal{O}_{X}=\mathscr{O}_{Y}$ and $f^{*}(A) \simeq L^{\otimes n_{2}}$. Thus, by Theorem 2.1, there is a semipositive continuous hermitian metric $k$ of $A$ such that $\left(f^{*}(A), f^{*}(k)\right) \simeq\left(L^{\otimes n_{2}}, h^{\otimes n_{2}}\right)$. Therefore, the assertion of the theorem follows from the previous observation.

\section{A variant of Theorem 0.2}

The following theorem is a consequence of Theorem 0.2 together with the arguments in [Zhang 1995, Theorem 3.3]. However, we can give a direct proof using ideas in the proof of Theorem 0.2 .

Theorem 4.1. Let $X$ be a projective algebraic variety over $\mathbb{C}$. Let L be an ample invertible sheaf on $X$ and let $h$ be a semipositive continuous hermitian metric of L. Let us fix a reduced subscheme $Y$ of $X, l \in H^{0}\left(Y,\left.L\right|_{Y}\right)$ and a positive number $\epsilon$. Then, for the given $X, L, h, Y, l$ and $\epsilon$, there is a positive integer $n_{1}$ such that, for all $n \geq n_{1}$, we can find $l^{\prime} \in H^{0}\left(X, L^{\otimes n}\right)$ with $\left.l^{\prime}\right|_{Y}=l^{\otimes n}$ and

$$
\sup \left\{h^{\otimes n}\left(l^{\prime}, l^{\prime}\right)(w) \mid w \in X(\mathbb{C})\right\} \leq e^{n \epsilon} \sup \{h(l, l)(w) \mid w \in Y(\mathbb{C})\}^{n} .
$$

Proof. In the case where $X$ is smooth over $\mathbb{C}$ and $h$ is smooth and positive, the assertion of the theorem follows from [Zhang 1995, Theorem 2.2], in which $Y$ is actually assumed to be a subvariety of $X$. However, the proof works well under the assumption that $Y$ is a reduced subscheme. First of all, let us see the theorem in the case where $X$ is smooth over $\mathbb{C}$ and $h$ is smooth and semipositive. As $L$ is ample, there is a positive smooth hermitian metric $t$ of $L$ with $t \leq h$. Let us choose a positive integer $m$ such that $e^{-\epsilon / 2} \leq(t / h)^{1 / m} \leq 1$ on $X(\mathbb{C})$. If we set $t_{m}=h^{1-1 / m} t^{1 / m}$, then $t_{m}$ is smooth and positive, so that, for a sufficiently large integer $n$, there is $l^{\prime} \in H^{0}\left(X, L^{\otimes n}\right)$ such that $\left.l^{\prime}\right|_{Y}=l^{\otimes n}$ and

$$
\sup \left\{t_{m}^{\otimes n}\left(l^{\prime}, l^{\prime}\right)(w) \mid w \in X(\mathbb{C})\right\} \leq e^{n \epsilon / 2} \sup \left\{t_{m}(l, l)(w) \mid w \in Y(\mathbb{C})\right\}^{n},
$$

and hence the assertion follows because $e^{-\epsilon / 2} h \leq t_{m} \leq h$ on $X(\mathbb{C})$.

For a general case, we use the same symbols $n_{0}, X \hookrightarrow \mathbb{P}^{N}, h_{\mathrm{FS}}, \phi, \omega$ and $\left\{\varphi_{i}\right\}$ as in the proof of Theorem 0.2. Clearly we may assume that $l \neq 0$. Since $L$ is ample, if $a_{0}$ is a sufficiently large integer, then, for each $j=0, \ldots, n_{0}-1$, there is 
$l_{j} \in H^{0}\left(X, L^{\otimes n_{0} a_{0}+j}\right)$ with $\left.l_{j}\right|_{Y}=l^{\otimes n_{0} a_{0}+j}$. Let us fix a positive number $A$ such that

$$
\sup \left\{h^{\otimes n_{0} a_{0}+j}\left(l_{j}, l_{j}\right)(w) \mid w \in X(\mathbb{C})\right\} \leq e^{A} \sup \{h(l, l)(w) \mid w \in Y(\mathbb{C})\}^{n_{0} a_{0}+j}
$$

for $j=0, \ldots, n_{0}-1$. We choose $i$ with $\left|\phi(x)-\varphi_{i}(x)\right| \leq \epsilon n_{0} / 2$ for all $x \in X$, and we set $h_{i}=\exp \left(-\varphi_{i}\right) h_{\mathrm{FS}}$. As $h_{i}$ is smooth and semipositive, for the given $\mathbb{P}^{N}, \mathcal{O}_{\mathbb{P}^{N}}(1)$, $h_{i}, Y, l^{\otimes n_{0}}$ (as an element of $H^{0}\left(Y,\left.\mathcal{O}_{\mathbb{P}^{N}}(1)\right|_{Y}\right)$ ) and $n_{0} \epsilon / 4$, there is a positive integer $a_{1}$ such that the assertion of the theorem holds for all $a \geq a_{1}$. We put

$$
n_{1}:=n_{0} \max \left\{a_{1}+a_{0}+1, \frac{4 A}{n_{0} \epsilon}-3 a_{0}+1\right\} .
$$

Let $n$ be an integer with $n \geq n_{1}$. If we set $n=n_{0}\left(a+a_{0}\right)+j\left(0 \leq j \leq n_{0}-1\right)$, then

$$
a \geq a_{1} \quad \text { and } \quad a \geq \frac{4 A}{n_{0} \epsilon}-4 a_{0},
$$

so that we can find $l^{\prime \prime} \in H^{0}\left(\mathbb{P}^{N}, \mathscr{O}_{\mathbb{P}^{N}}(a)\right)$ with $\left.l^{\prime \prime}\right|_{Y}=l^{\otimes n_{0} a}$ and

$$
\sup \left\{h_{i}^{\otimes a}\left(l^{\prime \prime}, l^{\prime \prime}\right)(w) \mid w \in \mathbb{P}^{N}(\mathbb{C})\right\} \leq e^{a\left(n_{0} \epsilon / 4\right)} \sup \left\{h_{i}\left(l^{\otimes n_{0}}, l^{\otimes n_{0}}\right)(w) \mid w \in Y(\mathbb{C})\right\}^{a},
$$

which implies that

$$
\sup \left\{h^{\otimes n_{0} a}\left(l^{\prime \prime}, l^{\prime \prime}\right)(w) \mid w \in X(\mathbb{C})\right\} \leq e^{(3 / 4) n_{0} a \epsilon} \sup \{h(l, l)(w) \mid w \in Y(\mathbb{C})\}^{n_{0} a},
$$

because of (3-1). Here we set $l^{\prime}=l^{\prime \prime} \otimes l_{j}$. Then, $\left.l^{\prime}\right|_{Y}=l^{\otimes n}$ and, using (4-1) and (4-2), we have

$$
\begin{aligned}
\sup \left\{h^{\otimes n}\right. & \left.\left(l^{\prime}, l^{\prime}\right)(w) \mid w \in X(\mathbb{C})\right\} \\
& \leq \sup \left\{h^{\otimes n_{0} a}\left(l^{\prime \prime}, l^{\prime \prime}\right)(w) \mid w \in X(\mathbb{C})\right\} \sup \left\{h^{\otimes n_{0} a_{0}+j}\left(l_{j}, l_{j}\right)(w) \mid w \in X(\mathbb{C})\right\} \\
& \leq e^{(3 / 4) n_{0} a \epsilon+A} \sup \{h(l, l)(w) \mid w \in Y(\mathbb{C})\}^{n},
\end{aligned}
$$

which implies the assertion because $(3 / 4) n_{0} a \epsilon+A \leq \epsilon n$.

\section{Arithmetic application}

As an application of Theorem 0.2, we have the following generalization of the arithmetic Nakai-Moishezon criterion (see [Zhang 1995, Corollary 4.8]).

Corollary 5.1. Let $\mathscr{X}$ be a projective and flat integral scheme over $\mathbb{Z}$. Let $\mathscr{L}$ be an invertible sheaf on $\mathscr{X}$ such that $\mathscr{L}$ is nef on every fiber of $\mathscr{X} \rightarrow \mathbb{Z}$. Let $h$ be an $F_{\infty}$-invariant semipositive continuous hermitian metric of $\mathscr{L}$, where $F_{\infty}$ is the complex conjugation map $\mathscr{X}(\mathbb{C}) \rightarrow \mathscr{X}(\mathbb{C})$. If $\widehat{\operatorname{deg}}\left(\hat{c}_{1}((\mathscr{L}, h) \mid \mathscr{Y})^{\operatorname{dim} \mathscr{Y}}\right)>0$ for all horizontal integral subschemes $\mathscr{Y}$ of $\mathscr{X}$, then, for an $F_{\infty}$-invariant continuous hermitian invertible sheaf $(\mathscr{M}, k)$ on $\mathscr{X}, H^{0}\left(\mathscr{X}, \mathscr{L}^{\otimes n} \otimes \mathscr{M}\right)$ has a basis consisting of strictly small sections for a sufficiently large integer $n$. 
Proof. Let $X$ be the generic fiber of $\mathscr{X} \rightarrow \operatorname{Spec}(\mathbb{Z})$ and let $Y$ be a subvariety of $X$. Let $\mathscr{Y}$ be the Zariski closure of $Y$ in $\mathscr{X}$. As

$$
\widehat{\operatorname{deg}}\left(\hat{c}_{1}\left(\left.(\mathscr{L}, h)\right|_{\mathscr{Y}}\right)^{\operatorname{dim} \mathscr{Y}}\right)>0,
$$

$\left.(\mathscr{L}, h)\right|_{\mathscr{Y}}$ is big by [Moriwaki 2012, Theorem 6.6.1], so that $H^{0}\left(\mathscr{Y},\left.\mathscr{L}^{\otimes n_{0}}\right|_{\mathscr{Y}}\right) \backslash\{0\}$ has a strictly small section for a sufficiently large integer $n_{0}$. Moreover, if we set $L=\left.\mathscr{L}\right|_{X}$, then $\left.L\right|_{Y}$ is big, and hence $\operatorname{deg}\left(L^{\operatorname{dim} Y} \cdot Y\right)>0$ because $L$ is nef. Therefore, $L$ is ample by the Nakai-Moishezon criterion for ampleness. In particular, by Theorem $0.2, h$ is semiample metrized. Thus the assertion follows from the arguments in [Zhang 1995, Theorem 4.2].

\section{Acknowledgements}

I would like to thank Professor Zhang and the referee for their comments and suggestions.

\section{References}

[Coman et al. 2013] D. Coman, V. Guedj, and A. Zeriahi, "Extension of plurisubharmonic functions with growth control”, J. Reine Angew. Math. 676 (2013), 33-49. MR 3028754 Zbl 1269.32018

[Demailly 1985] J.-P. Demailly, Mesures de Monge-Ampère et caractérisation géométrique des variétés algébriques affines, Mém. Soc. Math. France (N.S.) 19, 1985. MR 87g:32030 Zbl 0579.32012

[Fornæss and Narasimhan 1980] J. E. Fornæss and R. Narasimhan, "The Levi problem on complex spaces with singularities”, Math. Ann. 248:1 (1980), 47-72. MR 81f:32020 Zbl 0411.32011

[Moriwaki 2012] A. Moriwaki, "Zariski decompositions on arithmetic surfaces", Publ. Res. Inst. Math. Sci. 48:4 (2012), 799-898. MR 2999543 Zbl 1281.14017

[Zhang 1995] S. Zhang, "Positive line bundles on arithmetic varieties", J. Amer. Math. Soc. 8:1 (1995), 187-221. MR 95c:14020 Zbl 0861.14018

Communicated by Shou-Wu Zhang

Received 2014-11-09 Revised 2015-01-01 Accepted 2015-02-16

moriwaki@math.kyoto-u.ac.jp Department of Mathematics, Faculty of Science, Kyoto University, Kyoto 606-8502, Japan 


\section{Algebra \& Number Theory}

msp.org/ant

\section{EDITORS}

MANAGING EDITOR

Bjorn Poonen

Massachusetts Institute of Technology

Cambridge, USA

\author{
EDITORIAL BOARD CHAIR \\ David Eisenbud \\ University of California \\ Berkeley, USA
}

BOARD OF EDITORS

Georgia Benkart

Dave Benson

Richard E. Borcherds

John H. Coates

J-L. Colliot-Thélène

Brian D. Conrad

Hélène Esnault

Hubert Flenner

Edward Frenkel

Andrew Granville

Joseph Gubeladze

Roger Heath-Brown

Craig Huneke

János Kollár

Yuri Manin

Barry Mazur

Philippe Michel

Susan Montgomery
University of Wisconsin, Madison, USA

University of Aberdeen, Scotland

University of California, Berkeley, USA

University of Cambridge, UK

CNRS, Université Paris-Sud, France

University of Michigan, USA

Freie Universität Berlin, Germany

Ruhr-Universität, Germany

University of California, Berkeley, USA

Université de Montréal, Canada

San Francisco State University, USA

Oxford University, UK

University of Virginia, USA

Princeton University, USA

Northwestern University, USA

Harvard University, USA

École Polytechnique Fédérale de Lausanne

University of Southern California, USA
Shigefumi Mori

Raman Parimala

Jonathan Pila

Anand Pillay

Victor Reiner

Peter Sarnak

Joseph H. Silverman

Michael Singer

Vasudevan Srinivas

J. Toby Stafford

Richard Taylor

Ravi Vakil

Michel van den Bergh

Marie-France Vignéras

Kei-Ichi Watanabe

Efim Zelmanov

Shou-Wu Zhang
RIMS, Kyoto University, Japan

Emory University, USA

University of Oxford, UK

University of Notre Dame, USA

University of Minnesota, USA

Princeton University, USA

Brown University, USA

North Carolina State University, USA

Tata Inst. of Fund. Research, India

University of Michigan, USA

Harvard University, USA

Stanford University, USA

Hasselt University, Belgium

Université Paris VII, France

Nihon University, Japan

University of California, San Diego, USA

Princeton University, USA

\section{PRODUCTION}

production@msp.org

Silvio Levy, Scientific Editor

See inside back cover or msp.org/ant for submission instructions.

The subscription price for 2015 is US \$255/year for the electronic version, and $\$ 440 /$ year ( $\$ 55$, if shipping outside the US) for print and electronic. Subscriptions, requests for back issues and changes of subscribers address should be sent to MSP.

Algebra \& Number Theory (ISSN 1944-7833 electronic, 1937-0652 printed) at Mathematical Sciences Publishers, 798 Evans Hall \#3840, c/o University of California, Berkeley, CA 94720-3840 is published continuously online. Periodical rate postage paid at Berkeley, CA 94704, and additional mailing offices.

ANT peer review and production are managed by EditFLOW ${ }^{\circledR}$ from MSP.

\section{PUBLISHED BY}

7 mathematical sciences publishers

nonprofit scientific publishing

http://msp.org/

(C) 2015 Mathematical Sciences Publishers 


\section{Algebra \& Number Theory}

Volume $9 \quad$ No. $2 \quad 2015$

Lifting harmonic morphisms II: Tropical curves and metrized complexes

OMID AMINI, MATTHEW BAKER, ERWAN BRUgallé and JosePH RABINOFF

Noncommutative Hilbert modular symbols

IVAN HOROZOV

$p$-adic Hodge theory in rigid analytic families

REBECCA BELLOVIN

Semistable periods of finite slope families

RUOCHUAN LIU

ANAND DEOPURKAR and ANAND PATEL

Finite-dimensional quotients of Hecke algebras

493

IVAN LOSEV

Semiample invertible sheaves with semipositive continuous hermitian metrics

503 ATSUSHI MORIWAKI 